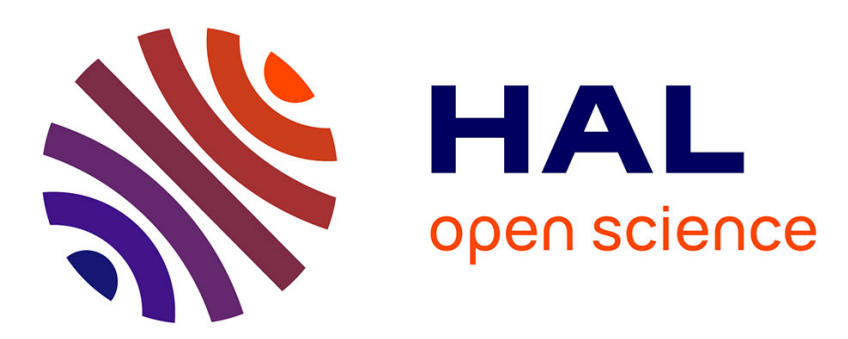

\title{
Anisothermal thermosetting resin cure monitored by optical fiber refractometer
}

Laurent Robert, Gilles Dusserre

\section{To cite this version:}

Laurent Robert, Gilles Dusserre. Anisothermal thermosetting resin cure monitored by optical fiber refractometer. Polymer Engineering and Science, 2014, 54 (3), p. 626-635. 10.1002/pen.23596 . hal-01611613

\section{HAL Id: hal-01611613 https://hal.science/hal-01611613}

Submitted on 8 Nov 2018

HAL is a multi-disciplinary open access archive for the deposit and dissemination of scientific research documents, whether they are published or not. The documents may come from teaching and research institutions in France or abroad, or from public or private research centers.
L'archive ouverte pluridisciplinaire HAL, est destinée au dépôt et à la diffusion de documents scientifiques de niveau recherche, publiés ou non, émanant des établissements d'enseignement et de recherche français ou étrangers, des laboratoires publics ou privés. 


\title{
Anisothermal Thermosetting Resin Cure Monitored by Optical Fiber Refractometer
}

\author{
Laurent Robert, Gilles Dusserre \\ Université de Toulouse; Mines Albi, INSA, UPS, ISAE; ICA (Institut Clément Ader), Campus Jarlard, \\ F-81013 Albi Cedex 09, France
}

\begin{abstract}
The cure monitoring of composite parts is an important key to optimize the industrial curing cycles. In this study, it is proposed to monitor the cure of a thermosetting resin by a Fresnel refractometer. The measurements are first compared to dielectric analyses and predictions of a thermo-kinetic model that uses parameters from the literature. Under isothermal conditions, the results show that the Fresnel power can be directly exploited to obtain qualitatively the degree of conversion. This is also demonstrated in anisothermal conditions by correcting the Fresnel signal from its temperature dependence. If the initial degree of conversion is known, its quantitative assessment is achieved using the Fresnel and temperature measurements.
\end{abstract}

\section{INTRODUCTION}

Thermosetting-based composite materials are widely used as structural materials because of their good ratio between weight and strength. However, some well-known issues concern damage [1], residual stresses [2, 3], or mechanical properties of these materials [4]. These issues meet some obstacles partly related to the variability of the properties induced by the manufacturing processes. Indeed, the quality of the composite part depends on the control of the process cycle and its parameters (temperature, pressure/vacuum, curing time). In order to improve the curing of thermosetting resins and therefore the quality and reliability of these composite parts, high performance in situ sensing systems are required for the monitoring of processes [5-16].

Many different techniques have been developed to characterize the cure process of thermosetting resins. One can cite (see, for example, Ref. 15] and references within) thermal measurements (such as differential scanning calorimetry (DSC) and thermal mechanical analysis (TMA)), dielectric analysis (DEA), nuclear magnetic resonance, dynamic mechanical analysis, ultrasonic techniques and optical measurements (by infrared or Raman spectroscopy, grating-based optical fiber sensors (OFS) and Fresnel refractometers). Among the methods cited above, mainly DEA and OFS methods are able to monitor the cure during the manufacturing process.

Further studies have demonstrated the advantages of OFS over conventional sensors: they are small and flexible, light, immune to electromagnetic interference, and can withstand corrosive environments and high temperatures. Most of the published works on monitoring of epoxy cross-linking for composite processes by OFS use a specific sensing element [5] or the Fresnel reflection. The Fresnel reflection at the end of an optical fiber has been reported in Refs. 8, 10, 11. 15, and 17, while other Fresnel devices have been also developed $[12,14]$. Another kind of refractive index OFS is based on in-fiber grating structures, e.g., long period gratings [16] or tilted fiber Bragg gratings [16, 18]. However, the sensitivity range is generally limited to refractive index below the refractive index of the optical fiber cladding. Optical-based sensors are in most cases calibrated by additional indirect measurements by DSC or TMA $[6,10,11,15]$. The drawback of this procedure, except the fact that both measurements are not done simultaneously, comes from the experimental conditions of DSC or TMA (size of the specimen, thermal environment) that are quite different from those encountered in more industrial situations. In this context, an interesting approach consists in using in the same experiment both heat flux sensors and OFS [17] for the determination of the degree of conversion.

Among the commercially available sensors in the context of industrial composite processes, the dielectric sensors allow in situ monitoring of the degree of cure. These sensors utilize the changes induced in the dielectric behavior of the resin to provide real-time and in situ information on the advancement of the process, generally also derived from some calibrations with the help of a quantitative analysis, for example, by DSC [9, 13, 19]. While advantages lie in its robustness, drawbacks come from the need of external calibration from DSC, and the size of the rigid sensors that provide only surface information.

Except methods based on heat flux measurements (DSC), all the devices described above are also sensitive 
to the temperature variations. Therefore, in cases of anisothermal curing, the calibration of the sensor needs to be temperature dependent. A supplementary appropriate evolution law is thus required for the measured quantity. For instance, in Ref. 20, the non-isothermal thermoset cure is monitored by an OFS, and the refractometer signal is expressed as the sum of two linear functions of temperature and chemical conversion.

Another way to determine the curing cycle of composites involves the modeling of the polymerization kinetics. For thermosetting resins, it is usually performed thanks to phenomenological models, requiring parameter identification from experimental data. In the case of epoxy/amine systems, Horie et al. [21] assumed an identical reactivity for primary and secondary amine functions leading to a model with a global reaction order of 3. A generalization of this model was proposed by Kamal and Sourour [22] including partial orders of catalytic and autocatalytic reactions to be identified, making the model very suitable for simulation of the curing of epoxy/amine systems. The main limitation stands for high degree of conversion when the reaction is mainly controlled by diffusion. This phenomenon can be taken into account by adding a diffusion factor, as proposed, for example, by Lam [23].

In this study we report on the cure monitoring of an epoxy resin suitable for liquid composite processes, by using simultaneously a multi-instrumentation based on an OFS (a Fresnel refractometer) and DEA. Two mold materials and sample thicknesses are used to enhance or reduce the exothermic phenomenon, providing isothermal and anisothermal curing data. The isothermal curing is exploited to correlate the optical signal, the dielectric signal, and the results of a kinetic modeling. It allows to base our analyses of the curing only on the signal from the weak-intrusive OFS. In the anisothermal case, an evolution law of the Fresnel power with temperature is proposed, based on the experimental results. Quantitative values of the degree of conversion are deduced from the experimental Fresnel signal by correcting it using the temperature changes. This approach is finally validated with a numerical model of the cure.

\section{EXPERIMENT}

\section{Dielectric Analysis}

Dielectric responses of thermoset resins are caused by the orientation of dipoles existing in functional groups and mainly (at low frequency or low temperature) by migration of ions present in epoxy resins due to their inherent impurities [24]. In a typical dielectric test, the resin sample is placed in contact with two electrodes of the dielectric sensor, and a sinusoidal voltage is applied to one electrode. The resulting sinusoidal current measured at the second electrode is attenuated in amplitude and shifted in phase in relation to the mobility of ions. One can show [24] that the ionic conductivity is proportional to the concentration of ions and to their mobility, which is a function of the (dynamic) resin viscosity. Therefore, the change in ion conductivity, related to the inverse of the ionic viscosity, can be used as an indicator of the curing state of resins and therefore composites. In this work, a 10-channel dielectric sensor system, DEA 230/10, from Netzsch Instruments was used. The sensors chosen for the experiments are single use sensors comprised of two interdigitated electrodes on a polyimide substrate. These sensors of size $10 \times 20 \mathrm{~mm}^{2}$ are simply placed in the mold in the vicinity of the resin. Dielectric measurements were conducted at various frequencies from $0.1 \mathrm{~Hz}$ to $10 \mathrm{kHz}$.

\section{Fresnel Refractometer}

An alternative approach makes use of the changes in the refractive index of a thermoset resin that occur during the curing due to resin shrinkage, by measuring the Fresnel reflection at the end face of an optical fiber $[6,10$, 11, 15-17]. For a typical epoxy resin, refractive index shows an increase of 0.05 for a fully cross-linked resin [17]. The principle of Fresnel reflection is briefly recalled: a light coming out from an optical source is launched into an optical fiber through a coupler. The tip of the optical fiber is cleaved very precisely and immersed into the resin. At the interface between the fiber core and the resin, there is an abrupt change in the refractive index. Since the fiber end face is cleaved perpendicular to its axis, the reflection coefficient is given by Fresnel laws. A small part of light that arrives is backreflected at the interface and passes again through the coupler and is detected by a photoreceiver. Its intensity depends directly on the resin refractive index. This technique has been demonstrated to be relevant to monitor the evolution of the cure processing [7, 8, 10, 15, 17]. Drawbacks come from the facts that this technique needs to be externally calibrated to give quantitative results and is not totally reproducible as the reflection coefficient depends on the quality of the optical fiber end cleavage. Note finally that temperature produces similar changes in the signal and needs to be compensated in cases of an anisothermal curing that can be a difficult task. In this study, the Fresnel reflection setup consists of a very stable laser source (Nettest ${ }^{\circledR}$ Tunics Plus CL) with an output power of $10 \mathrm{~mW}$ operated at $1550 \mathrm{~nm}$, connected to an optical circulator, and a high resolution Rifocs 575L power meter. An optical fiber pigtail of SMF28 was used, and attention was paid for the fiber optic end cut. The end face of the fiber is positioned in the mold in contact with the resin and in the vicinity of the dielectric sensor in cases of cross-correlation of both measurements. As there is no need for knowing the absolute value of the resin refractive index, the Fresnel reflected power is simply recorded versus time and is enough to monitor the relative evolution of the cure. 


\section{Materials}

The resin used in this work is the HexFlow ${ }^{\circledR}$ RTM6 from Hexcel ${ }^{\circledR}$ Composites, a premixed epoxy specifically dedicated to liquid composite processes, and commonly used in the manufacturing of composite reinforced by carbon fibers mainly in the aeronautic industry. In order to enhance or not the exothermal manifestation of the resin during its polymerization, two kinds of molds were used. A soft metallic (SM) mold, which was a $0.05 \mathrm{~mm}$ thick aluminum foil pan easily deformable, enabled good thermal transfer, containing $2.4 \mathrm{~mm}$ thick resin sample. A 1 $\mathrm{mm}$ thick silicon (SI) mold that exhibited low thermal conductivity was also used. In this case, the thickness of the resin sample was $12.8 \mathrm{~mm}$. These molds were simply placed on a steel plate in a thermally controlled oven (Vötsch VT4002). Type K micro-thermocouples were used with a Pico ${ }^{\circledR}$ TC-08 controller device to assess the temperature in the resin and the oven during all curing cycles.

\section{CURE MODELING}

This section details the equations used to calculate the degree of conversion $\alpha$ of the resin in order to compare our results to those of the literature. The RTM6 resin curing kinetics has been widely studied [17, 25-28], and reliable models are available in the literature. Let us consider a thin plane infinite volume whose thickness is $a$. This volume is occupied by a thermosetting resin of density $\rho$, specific heat $c_{P}$, thermal conductivity $k$, and conversion enthalpy $\Delta H_{T}$. The two faces exchange heat with the environment at temperature $T_{\text {env }}$ with a different heat exchange coefficient on the upper and the lower boundaries, respectively, $h_{\mathrm{u}}$ and $h_{1}$. The one-dimensional heat transfer equation given by Eq. 1 governs the resin temperature $T$ subjected to a source term proportional to the conversion rate.

$$
\rho c_{P} \frac{\partial T}{\partial t}=\nabla(k \nabla T)+\rho \Delta H_{T} \frac{\partial \alpha}{\partial t} .
$$

The heat flux $q_{\Gamma}$ exchanged with the environment through the boundaries $\Gamma$ gives

$$
q_{\Gamma \mathrm{u}, 1}=h_{\mathrm{u}, 1}\left(T-T_{\mathrm{env}}\right) \quad \text { on } \Gamma_{\mathrm{u}, 1}
$$

If the resin sample is thermally thin, $T$ can be assumed to be uniform in the thickness. The total heat energy balance in the volume delimited by an in-plane surface of area $A$ simplifies to $E q$. 3, by neglecting the heat conduction term:

$$
\begin{aligned}
& \rho c_{P} \frac{\partial T}{\partial t} a A=\rho \Delta H_{T} \frac{\partial \alpha}{\partial t} a A+2 h^{*}\left(T-T_{\text {env }}\right) A \\
& h^{*}=\frac{h_{\mathrm{u}}+h_{\mathrm{l}}}{2} .
\end{aligned}
$$

$T$ can easily be derived from $E q .3$ as a function of the conversion rate and environment temperature, by the following relationship:

$$
\frac{\partial T}{\partial t}=\frac{\Delta H_{T}}{c_{P}} \frac{\partial \alpha}{\partial t}+\frac{2 h^{*}}{a \cdot \rho \cdot c_{P}}\left(T-T_{\mathrm{env}}\right) .
$$

In $E q .4, \Delta H_{T}, c_{P}$, and $\rho$ are the material parameters whose values are reported by Lecointe [26]. $\Delta H_{T}$ is set to $414 \mathrm{~J} \cdot \mathrm{g}^{-1}$, other authors report similar values of 432 $\mathrm{J} \cdot \mathrm{g}^{-1}$ [17]. The specific heat capacity $c_{P}$ is assumed to be linearly dependent on the degree of conversion [29]. Thus, the step change around the vitrification/devitrification point is neglected. It has been verified by a parametric study that the results are not significantly affected by this assumption:

$$
c_{P}(\alpha, T)=c_{P}(\alpha=1, T) \alpha+c_{P}(\alpha=0, T)(1-\alpha) .
$$

The specific heat temperature dependence of the uncured and fully cured resins, respectively, $c_{P}(\alpha=0, T)$ and $c_{P}(\alpha=1, T)$, is fitted with a second-order polynomial as given in Ref. 29. The linear relationship between the specific volume and $\alpha$ established by Aduriz et al. [17] allows to calculate the resin density as a function of $\alpha$ :

$$
\rho(\alpha)=\rho(\alpha=1) \alpha+\rho(\alpha=0)(1-\alpha)
$$

using the density of the uncured and fully cured resin, respectively, $\rho(\alpha=0)=1117 \mathrm{~kg} \cdot \mathrm{m}^{-3}$ and $\rho(\alpha=1)=1141$ $\mathrm{kg} \cdot \mathrm{m}^{-3}$, reported in Ref. 29. In this approach, the thermal expansion is neglected. A modified Kamal-Sourour model [22] is used to calculate the conversion rate at each time step. A diffusion term has been added to the initial law in order to evaluate more accurately the slowed down conversion kinetic of the vitrified material [23]. The values of the coefficients of $E q .7$ used in the present work are taken from Refs. 25, 27, and 28 and given in Table $1 . R$ is the ideal gas constant.

$$
\left\{\begin{array}{l}
\frac{\partial \alpha}{\partial t}=\left(\frac{k_{1} k_{\mathrm{d}}}{k_{1}+k_{\mathrm{d}}}+\frac{k_{2} k_{\mathrm{d}}}{k_{2}+k_{\mathrm{d}}} \alpha^{m}\right)(1-\alpha)^{n} \\
k_{i}=A_{i} e^{-E_{i} / R T}, \quad i=1,2 \\
k_{\mathrm{d}}=A_{\mathrm{d}} e^{-E_{\mathrm{d}} / R T} e^{-b / f} \\
f=f_{0}+\delta\left(T-T_{\mathrm{g}}\right)
\end{array}\right.
$$

In order to take into account a diffusion mechanism in the modified Kamal-Sourour law, the glass transition temperature $T_{\mathrm{g}}$ of the resin must be evaluated as a function of $\alpha$. This is achieved by using the so-called DiBenedetto equation [30], Eq. 8, where the glass transition temperature $T_{\mathrm{g}}$ of the uncured and fully cured resin, respectively, $T_{\mathrm{g}, 0}=-15^{\circ} \mathrm{C}$ and $T_{\mathrm{g}, \infty}=220^{\circ} \mathrm{C}$, as well as the value of the adjustable coefficient $\lambda=0.2784$, is reported by Aduriz et al. [17]. 
TABLE 1. Values of the coefficients of the Kamal-Sourour model (Eq. 7).

\begin{tabular}{llllll}
\hline Coef. (unit) & $A_{1}\left(\mathrm{~min}^{-1}\right)$ & $E_{1}\left(\mathrm{~J} \cdot \mathrm{mol}^{-1}\right)$ & $A_{2}\left(\mathrm{~min}^{-1}\right)$ & $E_{2}\left(\mathrm{~J} \cdot \mathrm{mol}^{-1}\right)$ & $A_{\mathrm{d}}\left(\mathrm{J} \cdot \mathrm{mol}^{-1}\right)$ \\
\hline \multirow{2}{*}{ Coef. (unit) } & $4.50 \times 10^{6}$ & $7.47 \times 10^{4}$ & $1.30 \times 10^{6}$ & $5.84 \times 10^{4}$ & $1.42 \times 10^{28}$ \\
\hline & $\delta(-)$ & $f_{0}(-)$ & $b(-)$ & $m(-)$ & $n(-)$ \\
\hline
\end{tabular}

$$
T_{\mathrm{g}}=T_{\mathrm{g}, 0}+\frac{\left(T_{\mathrm{g}, \infty}-T_{\mathrm{g}, 0}\right) \lambda \alpha}{1-(1-\lambda) \alpha}
$$

A finite difference integration scheme is applied to $E q$. 4, coupled to Eqs. 5-8, in order to evaluate the resin temperature evolution with time when the thickness of the resin sample $a$, the initial temperature $T_{0}$, and the initial degree of conversion $\alpha_{0}$ are known, as well as the temperature of the environment versus time, $T_{\text {env }}(t)$, measured by a thermocouple located in the oven.

The exothermal source term related to resin cfonversion enthalpy leads to a resin temperature arising at a higher value than the environment. The value of this exothermal peak is directly related to the heat transfer coefficient between the resin and its environment, $h^{*}$. It is important to note that $h^{*}$ is actually an effective heat transfer coefficient that includes not only convection transfer effects, but also the contribution of the mold and the conductive transfer through the thickness of the resin. This parameter is the only unknown in the present model and depends on the experiment conditions, in particular on the thickness of the mold and the sample. It is proposed to identify the value of the coefficient $h^{*}$ by minimizing, using a Newton optimization algorithm, the gap between the measured and calculated maximum temperatures reached during the cure.

The value of $h^{*}$ has been identified for each experiment and allows to calculate the Biot number. It turns out that the assumption of uniform temperature field is only valid for the SM experiment (Biot number around 0.3). For the SI experiment, the large thickness of the sample involves a nonnegligible thermal gradient.

To summarize the material parameters being provided by the literature, the only data required to fully adjust the presented model is the resin maximal temperature. A validation of the applicability of the model to the experimental cases is obtained by comparing the calculated and measured resin temperatures versus time during the whole experiment. Once the validity of the simulation is established in "Isothermal cure monitoring assessment" section, the response of the model will be used as a reference in "Comparison to the model" section.

\section{RESULTS AND DISCUSSION}

In "Isothermal cure monitoring assessment" section, the responses of DEA and OFS are compared, allowing to analyze the curing comparatively to the results of the aforementioned model. In "Anisothermal cure monitoring assessment" section, it is experimentally demonstrated that $\alpha$ can be evaluated even in the case of anisothermal curing by correcting the Fresnel signal from its thermal variations. Finally, it is shown in "Comparison to the model" section that after an updating of the initial conditions, the model fits well the experimental results in anisothermal curing conditions.

\section{Isothermal Cure Monitoring Assessment}

Figure 1 shows the evolution of the ionic viscosity from the DEA, the Fresnel reflected power, and the thermocouple temperature measured in the vicinity of the other sensors during a thermal cycle. The cure is done at a relatively low temperature level $\left(140^{\circ} \mathrm{C}\right)$ in the SM mold with a thin resin sample in order to limit the temperature overshoot due to the exothermal reaction. The thermal cycle consists in an increase of temperature from $80^{\circ} \mathrm{C}$ to $140^{\circ} \mathrm{C}$ at a heating rate of $6^{\circ} \mathrm{C} \cdot \mathrm{min}^{-1}$ to reduce the temperature gradients (heating phase), followed by a cure plateau where the resin temperature remains almost constant. The third period is a simple cooldown by natural convection to ambient temperature (cooling phase).

We show clearly that the correlation between the dielectric and the optical signals is very good, for at least the two first phases. During the heating phase, the viscosity decreases until a minimum corresponding to the onset
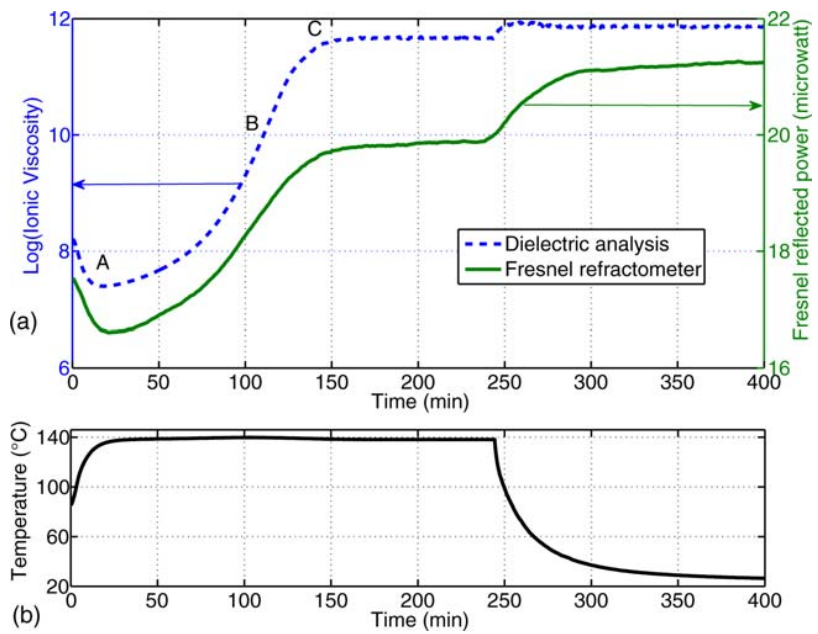

FIG. 1. Ionic viscosity and Fresnel reflected power (a) and temperature (b) evolution recorded for the whole temperature cycle applied to the resin sample (SM mold). [Color figure can be viewed in the online issue, which is available at wileyonlinelibrary.com.] 


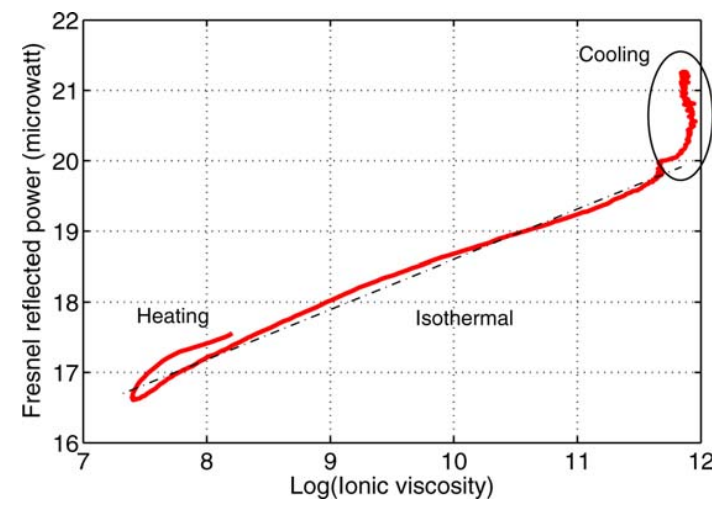

FIG. 2. Fresnel reflected power as a function of the ionic viscosity during the whole curing process (SM). [Color figure can be viewed in the online issue, which is available at wileyonlinelibrary.com.]

of cross-linking (point A in Fig. 1). The optical signal shows also a decrease since the refractive index is only influenced by the thermal changes. During the cure plateau, the cross-linking occurs (increase of the degree of conversion), involving an exothermal reaction which is dissipated by the metallic mold. These phenomena lead to an increase of the molar weight of the resin macromolecules and correspond physically to a densification of the resin network and shrinkage. It is manifested by an increase of the ionic viscosity in Fig. 1a. The reflected power follows the same evolution with time, and as an isothermal state can be considered, the increase in the reflected power is directly linked to the increase in the refractive index of the resin. It is noteworthy that the refractive index of the resin is proportional to the density and to the molecular weight of polymer repeat units following the Lorentz-Lorenz law [11]. It was also shown in Ref. 17 that there is a linear correlation between the resin refractive index and the degree of conversion $\alpha$. Furthermore, the relationship between the refractive index and the Fresnel reflected power can be considered, in a first approximation, as linear [11], because the amplitude of the refractive index variation is of $10^{-2}$ order that makes possible the linearization. This has been verified, as refractive index values ranging between 1.54 and 1.58 are reported in Ref. 17 for the RTM6 during its curing. It is thus concluded that the Fresnel power is a linear function of $\alpha$ at constant temperature, as also the ionic viscosity. After an inflection point of the DEA and Fresnel curves (point B in Fig. 1a), the attainment of the plateau of both signals correlates with the end of the cross-linking (point $\mathrm{C}$ in Fig. 1a). For the third phase (cooling), the dielectric and the optical signals differ because the ionic conductivity is slightly dependent on the temperature, and the sensor can be saturated by cured resin. On the contrary, the reflected power signal shows a monotonic function of the temperature as the polymerization reaction is finished.

In order to assess the correlation between the electric and optical signals, both are plotted altogether as depicted in Fig. 2. This figure shows that the better correlation between the two signals is get for the second phase (isothermal) and an almost linear correlation is found, demonstrating experimentally that the Fresnel refractometer can be used instead of the dielectric sensor, with all the advantages of OFS. In the first phase (heating), the correlation is also good whereas not so linear. Nevertheless, the minimum value of the ionic viscosity corresponds to the minimum value of the reflected power. Note that small differences between both signals can be attributed to the fact that the sensors are not exactly situated at the same location, and their spatial resolution is different. Finally, the dielectric sensor is unable to detect correctly the thermal variation of the cured resin during the cooling, and no correlation is seen in Fig. 2 (ellipse).

During the whole curing cycle, an analysis of the Fresnel reflected power can be done, as illustrated in Fig. 3. In the fist part of the curve, the resin moves from viscous to fluid, and the plot in Fig. 3 shows a decrease of the refractometer signal with the temperature, corresponding to a decrease of the refractive index with the temperature. During the cure phase, an increase of the optical signal from $16.6 \mu \mathrm{W}$ to $20 \mu \mathrm{W}$ is measured, whereas the temperature remains almost constant. In the cooling phase, as suggested by Giordano et al. [31] the glass transition temperature $\left(T_{\mathrm{g}}\right)$ can be evaluated by the inflection point in Fig. 3 during the cooling, around $110^{\circ} \mathrm{C}$, which is related to the change in the thermo-optic effect by crossing the glass transition zone.

As explained previously, the Fresnel reflection power is a linear function of $\alpha$ but cannot provide directly quantified values without calibration. A quantitative comparison of this experimental data to the degree of conversion is however possible by using the thermo-kinetic model presented in "Cure modeling" section. The model is adjusted to the actual experimental conditions by identifying the effective heat transfer coefficient only using the maximum temperature reached by the resin $\left(139.8^{\circ} \mathrm{C}\right.$ provided by the thermocouple). After convergence, a

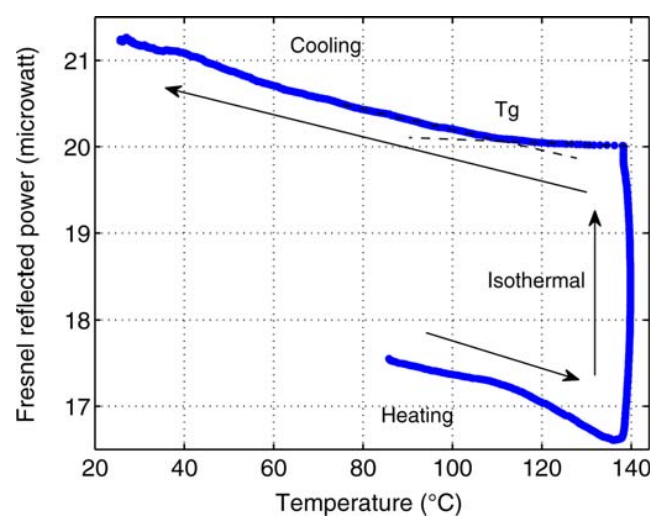

FIG. 3. Fresnel refractometer signal as a function of the thermocouple temperature during the whole curing process (SM). [Color figure can be viewed in the online issue, which is available at wileyonlinelibrary.com.] 


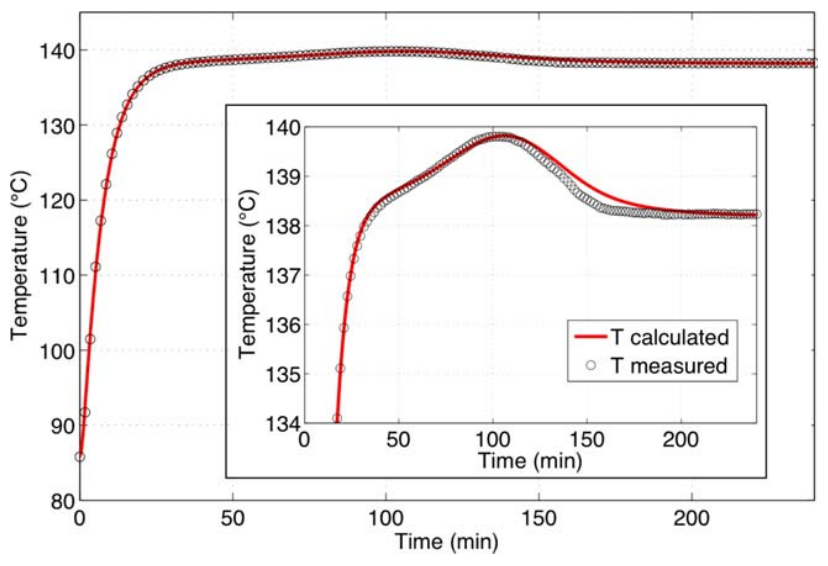

FIG. 4. Measured and calculated resin temperatures as functions of time during the curing process in the SM mold. A detailed view in the $134-140^{\circ} \mathrm{C}$ temperature range is inserted. [Color figure can be viewed in the online issue, which is available at wileyonlinelibrary.com.]

reasonable value of $h^{*}=65 \mathrm{~W} \cdot \mathrm{m}^{-2} \cdot \mathrm{K}^{-1}$ is found. To analyze the results, we first focus on the temperature evolution versus time. Figure 4 shows that the results of the model fit almost perfectly to the whole experimental curve, ensuring the relevance of the model. A maximum deviation of $1.4^{\circ} \mathrm{C}$ is found in the temperature increase, but the most significant bias is located just after the weak exothermal manifestation (bias lower than $0.3^{\circ} \mathrm{C}$ in cure plateau), as can be seen in the inserted plot in Fig. 4.

The model also provides the evolution of the conversion degree $\alpha$ which is plotted versus the Fresnel reflected power in Fig. 5. As expected, in the almost isothermal condition involved in this case, a first rough analysis shows that the Fresnel reflected power is a linear function of $\alpha$. In detail, before the onset of polymerization, the decrease of the Fresnel reflected power is directly linked to the thermal sensitivity of the signal. Moreover, the small deviation to the linearity at the end of the cure can be associated to an overestimation of the calculated temperature after the exothermic temperature peak, from about 120 to $180 \mathrm{~min}$. It leads to an overestimation of $\alpha$

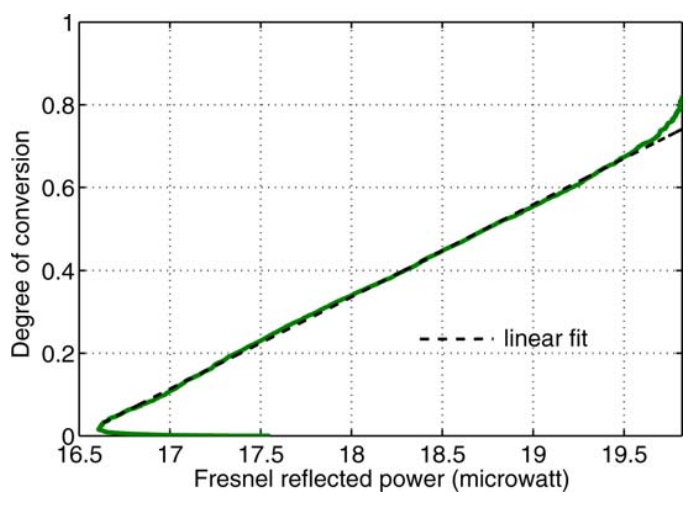

FIG. 5. Calculated degree of conversion plotted versus Fresnel reflected power during the curing process in the SM mold. [Color figure can be viewed in the online issue, which is available at wileyonlinelibrary.com.]

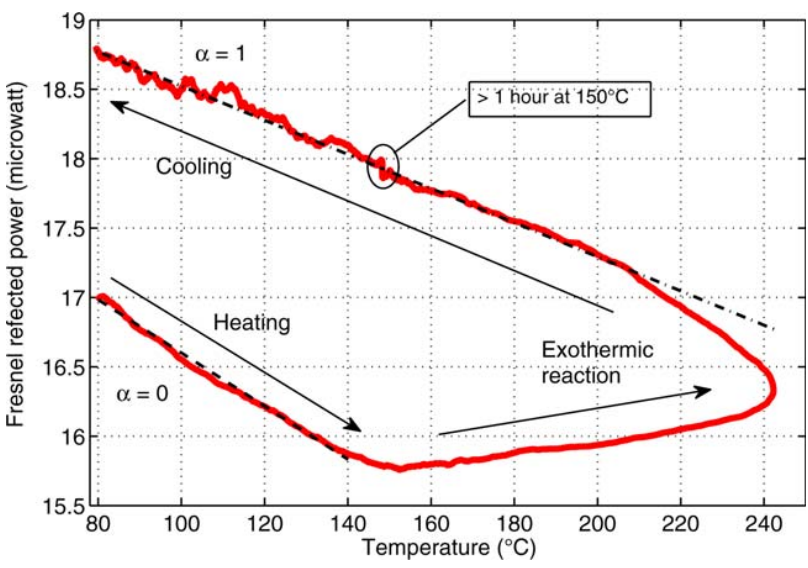

FIG. 6. Fresnel reflected power as a function of the thermocouple temperature during the whole curing cycle (SI mold). [Color figure can be viewed in the online issue, which is available at wileyonlinelibrary.com.]

given by the model. The model also provides an estimation of the evolution of $T_{\mathrm{g}}$ during the curing process. The final value at the end of the cure reaches $127^{\circ} \mathrm{C}$ which is necessarily also overestimated. This is however consistent with the glass transition manifestation deduced from the Fresnel power curve in Fig. 3, which exhibits a slope change around $110^{\circ} \mathrm{C}$.

\section{Anisothermal Cure Monitoring Assessment}

Another curing experience has been conducted in the insulating SI mold with a substantial thick resin sample, allowing large exothermal manifestations and an anisothermal cure. Note that before this experiment, the RTM6 sample has experienced an inadvertent thermal exposure suspected to have slightly modified the initial degree of cure. The thermal cycle consists in an increase of temperature from $80^{\circ} \mathrm{C}$ to $150^{\circ} \mathrm{C}$ at a heating rate of $6^{\circ} \mathrm{C} \cdot \mathrm{min}^{-1}$, followed by a cure plateau where the temperature setting stands at $150^{\circ} \mathrm{C}$, and then by a cooldown by natural convection to room temperature. Figure 6 shows the evolution of the Fresnel reflected power as a function of the thermocouple temperature during the whole cycle. During the heating step, the Fresnel power reflected in the uncured resin $(\alpha=0)$ decreases linearly with the temperature up to $140^{\circ} \mathrm{C}$ when the exothermal reaction begins to occur. During the exothermal polymerization (the resin temperature is above $150^{\circ} \mathrm{C}$ during about $50 \mathrm{~min}$ ), both temperature and $\alpha$ evolve. This results in a soft increase of the Fresnel power. Contrarily to the curing in the SM mold, as expected it was observed a huge overshoot of the temperature which corresponds to the exothermal manifestation of the chemical bonding: the temperature reaches more than $240^{\circ} \mathrm{C}$. It is explained because the diffusion of the temperature was not at all facilitated in the SI mold, and only low heat flux was able to be exchanged between the sample and the mold. The large thermal exposure undergone by the resin allows to 


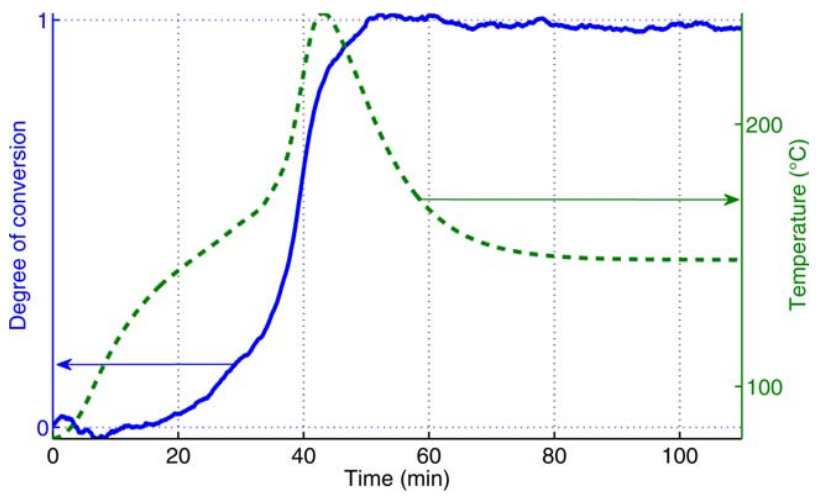

FIG. 7. Experimental degree of conversion and temperature evolution during the curing process (SI). [Color figure can be viewed in the online issue, which is available at wileyonlinelibrary.com.]

assume that the polymerization was fully completed in the linear part of the temperature decrease (below $210^{\circ} \mathrm{C}$ ). This is consistent with the fact that almost no evolution of the Fresnel power was observed when the resin temperature was maintained at the temperature setting during about $1 \mathrm{~h}$ (see ellipse in Fig. 6).

It was shown in "Isothermal cure monitoring assessment" section that the Fresnel power $P$ is an affine function of $\alpha$. The Fresnel power also evolves linearly with the temperature so that $P$ can be written as:

$$
P(\alpha, T)=P(\alpha=1, T) \alpha+P(\alpha=0, T)(1-\alpha) .
$$

Both terms $P(\alpha=0, T)$ and $P(\alpha=1, T)$ are deduced from the experimental data presented in Fig. 6 by fitting an affine function represented, respectively, by the dashed and dot-dashed lines in this figure. By inverting Eq. 9, the temporal evolution of $\alpha$ can be easily deduced from the experimental curve $P(\alpha, T)$ in Fig. 6 . The results are plotted in Fig. 7 together with the measured temperature. It is clearly shown that the plot of $\alpha$ is consistent with the temperature evolution. The measured degree of conversion oscillates around zero up to a temperature about $125^{\circ} \mathrm{C}$. At this time $(15 \mathrm{~min})$, the polymerization starts and the conversion rate accelerates. After the exothermal peak, the temperature decreases while $\alpha$ still increases more slowly until it reaches its maximal value, 1 . The signal then oscillates around this last value during the cooling phase due to noise in the Fresnel signal (see also Fig. 6) without any physical meaning.

\section{Comparison to the Model}

It was shown in the previous section that the Fresnel reflected power provides an evaluation of $\alpha$ even under anisothermal conditions. In order to assess the relevance of $\alpha$ measured by this way, a comparison to a model is also proposed. However, the model described in "Cure modeling" section is not relevant because of the thermal gradients. For that purpose, a two-dimensional (2D) finite element numerical simulation has been achieved in order to take into account the temperature gradient through the thickness of the sample. The model includes the $1 \mathrm{~mm}$ thick SI mold (conductivity $0.23 \mathrm{~W} \cdot \mathrm{m}^{-1} \cdot \mathrm{K}^{-1}$, density $1200 \mathrm{~kg} \cdot \mathrm{m}^{-3}$, and specific heat $1590 \mathrm{~J} \cdot \mathrm{kg}^{-1} \cdot \mathrm{K}^{-1}$ ) and the $12.8 \mathrm{~mm}$ thick RTM6 sample (conductivity ranging from 0.1 to $0.23 \mathrm{~W} \cdot \mathrm{m}^{-1} \cdot \mathrm{K}^{-1}$ and expressed as a function of $\alpha$, according to Ref. 26). The model solves the $2 \mathrm{D}$ heat transfer equation Eq. 1 coupled to Eqs. 5-8. Boundary conditions are temperature and flux continuity at the mold/sample interface and convective heat transfer between the external boundaries and the environment at $T_{\text {env }}$ controlled by the heat transfer coefficient $h$. Figure 8a summarizes the finite element modeling and the meshes. Figure $8 \mathrm{~b}$ and $\mathrm{c}$ presents, respectively, the temperature field and the degree of conversion through the thickness at the exothermal peak for which the gradient is maximal. The temperature deviation exceeds more than $30^{\circ} \mathrm{C}$, and the degree of conversion ranges from $71 \%$ to $94 \%$, requiring to consider accurately the actual sensor location. Figure $8 \mathrm{~b}$ also locates the thermocouple and the Fresnel sensor in the thickness of the resin sample. The values extracted at points $\mathrm{A}$ and $\mathrm{B}$ in Fig. $8 \mathrm{~b}$ (respectively, $\mathrm{C}$ and $\mathrm{D}$ ) are used to compare FEA results to the experimental temperature (respectively, Fresnel signal) in order to take into account the sensor location uncertainties.

As previously conducted in "Isothermal cure monitoring assessment" section, the heat exchange coefficient $h$ was identified by adjusting the maximum experimental temperature of the resin (i.e., at the exothermal peak) to the simulated one at points $\mathrm{A}$ and $\mathrm{B}$. The optimized value found for $h$ is $45 \mathrm{~W} \cdot \mathrm{m}^{-2} \cdot \mathrm{K}^{-1}$, a relevant order of magnitude for natural convection in air. In Fig. 9a are plotted the FEA results (degree of conversion) at both points $C$ and $\mathrm{D}$ and in Fig. 9b the FEA results (temperature) at both points A and B. Despite large gradients observed in the SI curing experiment, small discrepancies are finally noticed in the vicinity of both sensors. Figure 9a also compares the experimental and calculated conversion kinetics with the assumption that the initial degree of conversion is zero. It is shown that both curves agree qualitatively. However, it turns out that the model is delayed of about $14 \mathrm{~min}$. This is obviously related to the delay observed also between the experimental and calculated temperatures (see Fig. 9b). In our opinion, this discrepancy is necessarily related to a nonzero initial degree of conversion of the RTM6 sample at the beginning of this experiment, as mentioned previously. For that reason, the initial value of the degree of conversion $\alpha_{0}$ was also identified to adjust the experimental and calculated time-temperature data. The last results are plotted in Fig. 10. As shown in Fig. 10b, a very proper agreement is obtained with a reasonable value of the initial degree of conversion $\alpha_{0}=6.25 \%$, explained by a long exposure to the initial temperature before to conduct the SI experience. Naturally, the Fresnel data need to be processed also using 


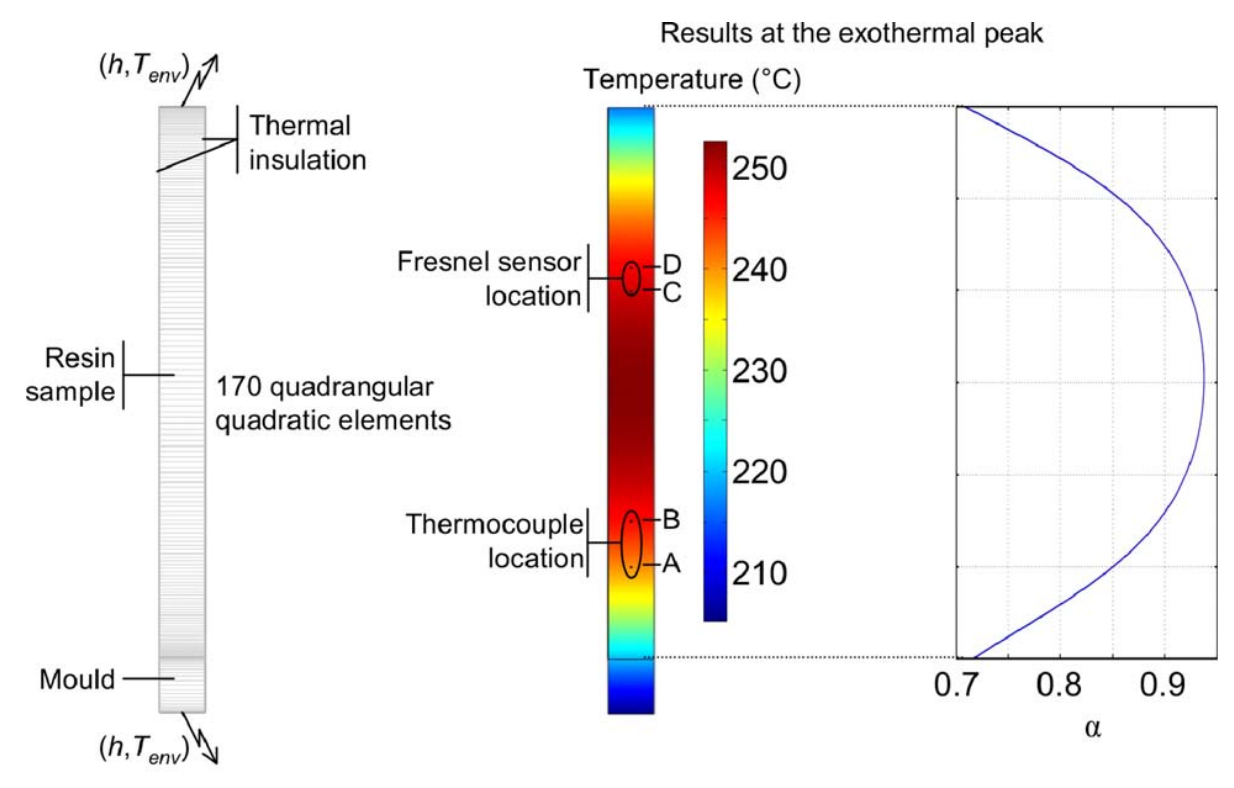

(a) (b) (c)

FIG. 8. Finite element analysis. (a) FEA model, meshes, and boundary conditions. Results at the exothermal peak: (b) temperature and (c) degree of conversion through the thickness of the sample. The locations of the sensors are also presented in this figure. [Color figure can be viewed in the online issue, which is available at wileyonlinelibrary.com.]

this value of $\alpha_{0}$. This is achieved using the following equation:

$$
P(\alpha, T)=P(\alpha=1, T) \frac{\alpha_{0}-\alpha}{\alpha_{0}-1}+P\left(\alpha=\alpha_{0}, T\right) \frac{1-\alpha}{1-\alpha_{0}},
$$

where $P\left(\alpha=\alpha_{0}, T\right)$ is fitted from Fig. 6 using the linear part of the curve before the onset of polymerization. It is shown in Fig. 10a that a good quantitative agreement is obtained between the measured and calculated $\alpha$ using these initial conditions. Both temperature and degree of conversion evolution are very properly described by the model.
It was thus demonstrated that an experimental evaluation of the degree of conversion of a thermosetting resin could be obtained using a Fresnel refractometer even under anisothermal conditions. An absolute value of the degree of conversion is available if the measurement is calibrated. An in situ calibration was performed using the evolution with temperature of the measured Fresnel power for two different constant degrees of conversion. The initial degree of conversion is constant up to the onset of polymerization. A first calibration set of data is then available at the beginning of the experiment if the initial

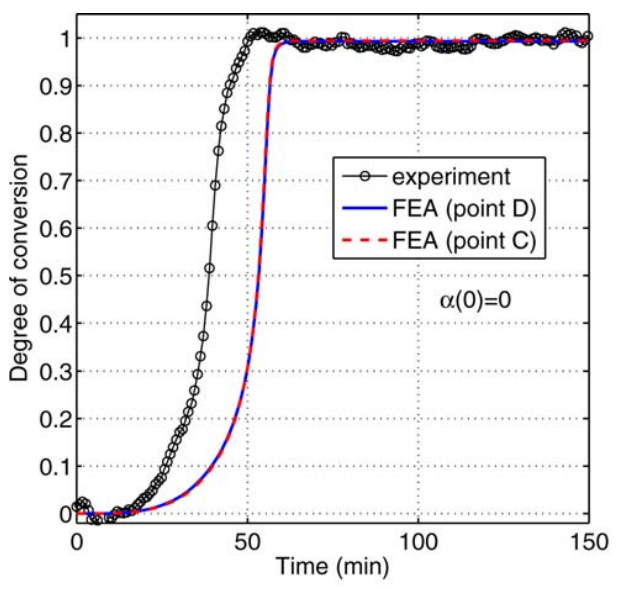

(a)

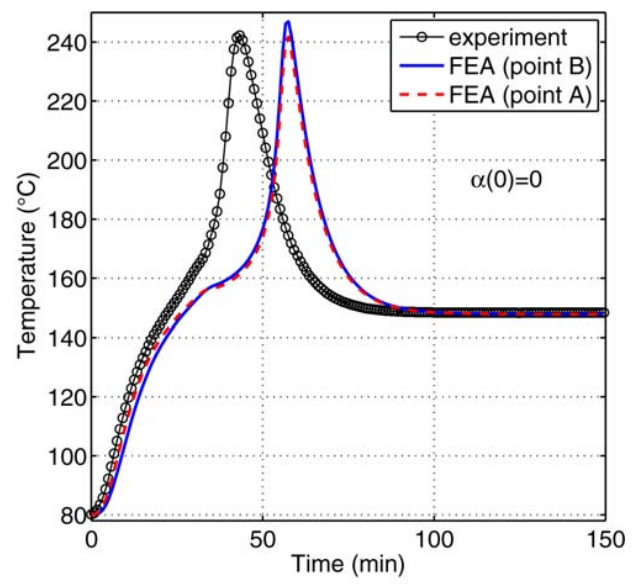

(b)

FIG. 9. Comparison of experimental and computed data with a zero initial degree of conversion: (a) degree of conversion and (b) temperature (SI mold). [Color figure can be viewed in the online issue, which is available at wileyonlinelibrary.com.] 


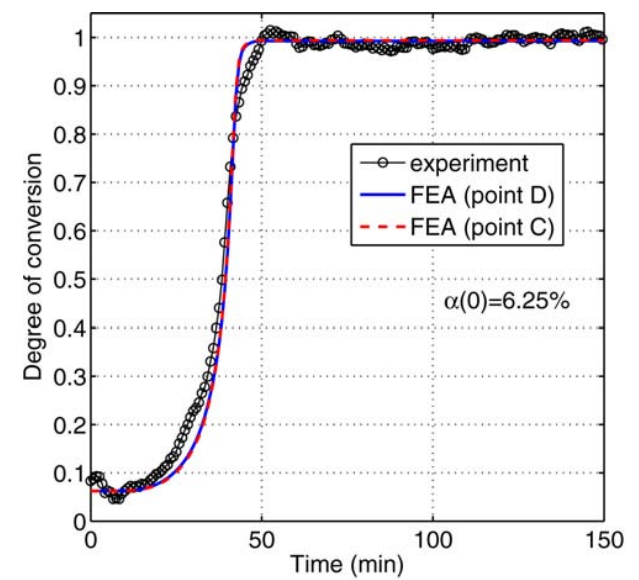

(a)

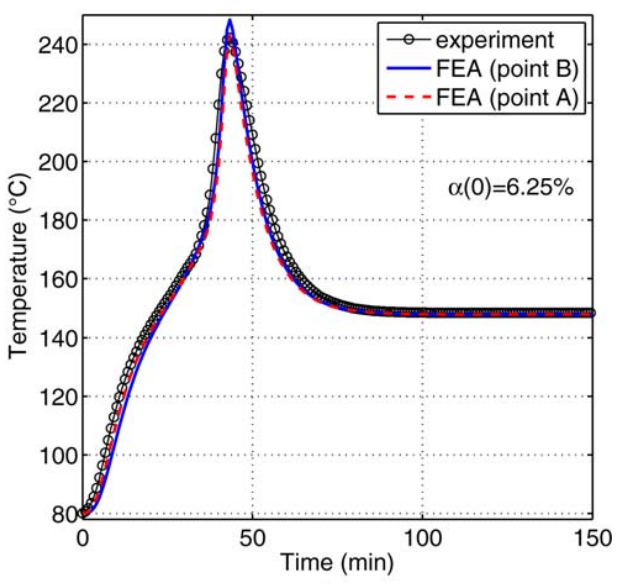

(b)

FIG. 10. Comparison of experimental and computed data with an optimized initial degree of conversion: (a) degree of conversion and (b) temperature (SI mold). [Color figure can be viewed in the online issue, which is available at wileyonlinelibrary.com.]

degree of conversion is known. The second set of data available for the calibration of the measurement is obtained at the end of the experiment, when the resin is fully polymerized. The cooldown step then provides the variation of the Fresnel power as a function of temperature for a constant $\alpha$. If the initial degree of conversion is known, this method provides a posteriori an absolute value of $\alpha$ once the polymerization has ended. It could also be used in real time to obtain a relative value of $\alpha$ and then monitor the process. To enhance the method and obtain an absolute value of $\alpha$, it is mandatory to calibrate the setup a priori by monitoring a cure of the resin during a cycle with high temperature, as performed here in the SI mold. Nevertheless, it is not sure that the cleaning of the fiber end after calibration does not affect the cleaved surface and thus the refraction of the light. The second way to provide a priori an absolute value is to ensure a perfect cleavage of the fiber end allowing to determine the refraction index of the resin from the Fresnel power. If a reproducible evolution of the refraction index with temperature and degree of conversion could be available, an absolute value of the degree of conversion would be provided directly from the Fresnel power measurement.

\section{SUMMARY AND CONCLUSIONS}

In this paper, the cure of the RTM6 thermosetting resin was investigated using a thermocouple and an optical fiber Fresnel refractometer. The quasi-perfect correlation between the optical signal and the signal from a commercial DEA intended to the composite manufacturing industry was first experimentally demonstrated. It allowed to base our analyses only on the Fresnel power and temperature evolutions. Two kinds of mold materials and sample thicknesses were used to enhance or reduce the exothermic phenomenon. A cure simulation was also performed in order to compare it to our experimental results. Two assumptions have been made: for thin samples, temperature gradient is neglected and a semi-analytical model is relevant, whereas gradients have been taken into account for thick samples thanks to a finite element analysis. For the isothermal cure, it was shown that the Fresnel signal evolves linearly with the calculated degree of conversion, and a perfect agreement was achieved for the whole temperature curves. In the case of anisothermal cure caused by a confined exothermic reaction, the degree of conversion was experimentally found by correcting the Fresnel power from the temperature variations, provided that the initial and final degrees of conversion are known. A comparison to the FEA model showed a mismatch with the experimental results, which has been cancelled out by adjusting the initial degree of conversion. An absolute value of the degree of conversion is thus provided a posteriori once the polymerization has ended. This approach can be directly applied in real time to obtain a relative value of the degree of conversion of a composite part instrumented by Fresnel and temperature sensors, during the manufacturing process.

\section{ACKNOWLEDGMENTS}

The authors are grateful for the financial support by the French Ministry of the Economy, Finance and Industry in the framework of the Carnot M.I.N.E.S. Institute and acknowledge $\mathrm{C}$. Vergnes for his great contribution to the experimental work.

\section{REFERENCES}

1. N. Takeda and S. Ogihara, Compos. Sci. Technol., 52, 309 (1994).

2. F. Colpo, L. Humbert, and J. Botsis, Compos. Sci. Technol., 67, 1830 (2007). 
3. M. Mulle, F. Collombet, P. Olivier, R. Zitoune, C. Huchette, F. Laurin, and Y.H. Grunevald, Compos. Part A, 40, 1534 (2009).

4. M. Mulle, R. Zitoune, F. Collombet, L. Robert, and Y.H. Grunevald, Compos. Struct., 91, 48 (2009).

5. M.A. Afromowitz, J. Lightwave Technol., 6, 1591 (1988).

6. J.S. Kim and D.G. Lee, J. Compos. Mater., 30, 1436 (1996).

7. P.A. Crosby, G.R. Powell, G.F. Fernando, C.M. France, R.C. Spooncer, and D.N. Waters, Smart Mater. Struct., 5, 415 (1996).

8. Y.M. Liu, C. Ganesh, J.P.H. Steele, and J.E. Jones, J. Compos. Mater., 31, 87 (1997).

9. M.J. O'Dwyer, G.M. Maistros, S.W. James, R.P. Tatam, and I.K. Partridge, Meas. Sci. Technol., 9, 1153 (1998).

10. A. Cusano, G. Breglio, M. Giordano, A. Calabro, and A. Cutolo, Sens. Actuators A, 84, 270 (2000).

11. M. Giordano, L. Nicolais, A.M. Calabro, S. Cantoni, A. Cusano, G. Breglio, and A. Cutolo, Polym. Compos., 21, 523 (2000).

12. E. Chailleux, M. Salvia, N. Jaffrezic-Renault, V. Matejee, and I. Kasik, Smart Mater. Struct., 10, 194 (2001).

13. H.G. Kim and D.G. Lee, Compos. Struct., 57, 91 (2002).

14. C. Li, M. Cao, R. Wang, Z. Wang, Y. Qiao, L. Wan, Q. Tian, H. Liu, D. Zhang, T. Liang, and C. Tang, Compos. Sci. Technol., 63, 1749 (2003).

15. S. Vacher, J. Molimard, H. Gagnaire, and A. Vautrin, Polym. Polym. Compos., 12, 269 (2004).

16. S.J. Buggy, E. Chehura, S.W. James, and R.P. Tatam, J. Opt. A, 9, 60 (2007).

17. X.A. Aduriz, C. Lupi, N. Boyard, J.L. Bailleul, D. Leduc, V. Sobotka, N. Lefèvre, X. Chapeleau, C. Boisrobert, and D. Delaunay, Compos. Sci. Technol., 67, 3196 (2007).
18. G. Laffont and P. Ferdinand, Meas. Sci. Technol., 1, 765 (2001).

19. D.G. Lee and H.G. Kim, J. Compos. Mater., 38, 977 (2004).

20. V. Antonucci, M. Giordano, A. Cusano, J. Nasser, and L. Nicolais, Compos. Sci. Technol., 66, 3273 (2006).

21. K. Horie, H. Hiura, M. Sawada, I. Mita, and H. Kambe, J. Polym. Sci. Part A-1, 8, 1357 (1970).

22. M.R. Kamal and S. Sourour, Polym. Eng. Sci., 13, 59 (1973).

23. P.W.K. Lam, Polym. Compos., 8, 427 (1987).

24. J. Mijovic, J.M. Kenny, A. Maffezzoli, A. Trivisano, F. Bellucci, and L. Nicolais, Compos. Sci. Technol., 49, 277 (1993).

25. P.I. Karkanas and I.K. Partridge, Polym. Int., 41, 183 (1996).

26. D. Lecointe, "Caractérisation, simulation des processus de transferts lors d'injection de résine pour le procédé RTM," PhD Thesis, Univ. de Nantes, France (1999) (in French).

27. P.I. Karkanas and I.K. Partridge, J. Appl. Polym. Sci., 77, 1419 (2000).

28. P.I. Karkanas and I.K. Partridge, J. Appl. Polym. Sci., 77, 2178 (2000)

29. D. Lecointe, M. Villière, S. Nakouzi, V. Sobotka, N. Boyard, F. Schmidt, and D. Delaunay, Key Eng. Mater., 504, 1091 (2012).

30. J.P. Pascault, H. Sautereau, J. Verdu, and R.J.J. Williams, Thermosetting Polymer, Marcel Dekker, New York (2002).

31. M. Giordano, A. Laudati, M. Russo, J. Nasser, G.V. Persiano, and A. Cusano, Thin Solid Films, 450, 191 (2004). 